\title{
Polymers for Biotechnology and Bioengineering
}

\author{
Lisa Brannon-Peppas \\ Biogel Technology, Inc.
}

While many of the early biomaterials had their origins in non-medical applications, the polymers being developed today as biomaterials are targeted quite specifically for biological use. These materials are designed for particular uses, whether that be hard tissue replacement, organ replacement, controlled drug delivery or other applications. Some materials are designed for extended stability and structural integrity, while in contrast others are built to degrade within extremely short time periods. In some cases, the biomaterial must be virtually invisible to the body, and in other cases the material is extremely sensitive to the biological environment and will change its structure and behavior depending upon changes it senses within the body.

Some of the most exciting areas ofbiomaterials research where significant strides are currently being made in specialized polymer synthesis and polymer engineering for advanced biomedical applications include:

- Biodegradable polymers as scaffolds for tissue engineering,

- Bioresponsive hydrogels for controlled drug delivery and biosensors,

- Hydrolytically degradable biomaterials in treatment of cancers, and

- Fabrication of structurally-specific biomaterials on the molecular level using microfabrication techniques.

This review paper will address briefly the past methods used to develop biomaterials and will concentrate on the advances being made in the areas of controlled drug delivery, tissue engineering, biodegradable biomaterials and environmental y responsive biomaterials. A range of materials will be discussed, including hydrogels and poly(lactic-co-glycolic acid)s. Novel formulations which include composite polymer structures of biodegradable and non-degradable biomaterials for use in controlled drug delivery will also be presented. 